\title{
New algorithm for integration between wireless microwave sensor network and radar for improved rainfall measurement and mapping
}

\author{
Y. Liberman ${ }^{1}$, R. Samuels ${ }^{2}$, P. Alpert ${ }^{2}$, and H. Messer ${ }^{1}$ \\ ${ }^{1}$ Tel Aviv University, School Of Electrical Engineering, Tel Aviv 6997801, Israel \\ ${ }^{2}$ Tel Aviv University, Porter School for Environmental Studies, Tel Aviv 6997801, Israel \\ Correspondence to: Y. Liberman (yoavliberman@mail.tau.ac.il)
}

Received: 28 December 2013 - Published in Atmos. Meas. Tech. Discuss.: 6 May 2014

Revised: 9 August 2014 - Accepted: 20 August 2014 - Published: 17 October 2014

\begin{abstract}
One of the main challenges for meteorological and hydrological modelling is accurate rainfall measurement and mapping across time and space. To date, the most effective methods for large-scale rainfall estimates are radar, satellites, and, more recently, received signal level (RSL) measurements derived from commercial microwave networks (CMNs). While these methods provide improved spatial resolution over traditional rain gauges, they have their limitations as well. For example, wireless CMNs, which are comprised of microwave links (ML), are dependant upon existing infrastructure and the ML' arbitrary distribution in space. Radar, on the other hand, is known in its limitation for accurately estimating rainfall in urban regions, clutter areas and distant locations. In this paper the pros and cons of the radar and ML methods are considered in order to develop a new algorithm for improving rainfall measurement and mapping, which is based on data fusion of the different sources. The integration is based on an optimal weighted average of the two data sets, taking into account location, number of links, rainfall intensity and time step. Our results indicate that, by using the proposed new method, we not only generate more accurate 2-D rainfall reconstructions, compared with actual rain intensities in space, but also the reconstructed maps are extended to the maximum coverage area. By inspecting three significant rain events, we show that our method outperforms CMNs or the radar alone in rain rate estimation, almost uniformly, both for instantaneous spatial measurements, as well as in calculating total accumulated rainfall. These new improved 2-D rainfall maps, as well as the accurate rainfall measurements over large areas at sub-hourly timescales, will allow for improved understanding, initialization, and calibra-
\end{abstract}

tion of hydrological and meteorological models mainly necessary for water resource management and planning.

\section{Introduction}

The need for reliable, high-resolution rainfall measurement and mapping is increasing, as such data are the principle drivers for hydrometeorological models, climate studies, urban planning and flood warning systems. Current methods such as rain gauges, radar, microwave links (ML), and even satellites can provide measurements, yet the ability to generate high-resolution maps from them is limited. Rain gauges, which provide the most reliable estimates, are limited due to their point location measurements, which cannot provide accurate spatial estimates, especially in areas of complex topography or high spatial variability (Rayitsfeld et al., 2012).

Other methods which have been adopted to overcome this spatial challenge, include radar estimates and, more recently, measurements from wireless ML networks (Messer et al., 2006). Naturally, due to both environment and technological limitations, the estimates from such sources may have high levels of uncertainty and errors (e.g. Mackenzie et al., 1993). For meteorologists and hydrologists attempting to use this information to either better understand storm dynamics or to inform infrastructure planning, each of these methods has unique information. Given this plethora of data, it has recently been acknowledged that precipitation estimates with a spatial and temporal resolution of $4 \mathrm{~km}$ and $30 \mathrm{~min}$, respectively, are realistic target levels useful for many researches and applications (Sorooshian et al., 2011). This is particularly true for estimation of orographic rainfall distribution 
on the high-meso-gamma-scale resolution, as reviewed by Alpert et al. (1994). Unfortunately, the formats of the data, as well as the varying scales and limitation on their availability, make it difficult to use them in a complementary way.

In this study we present a new technique for integrating between radar and commercial microwave networks (CMNs) data in order to improve the accuracy and reliability of rainfall estimates. The integration of multiple sources allows for weighing the estimates appropriately in line with the advantages and disadvantages of the multiple rain sources included. This design leaves room for the incorporation of other data sources (e.g. satellites) in the future.

Furthermore, this paper also demonstrates how the integrative approach provides better instantaneous as well as cumulative rainfall estimates, both spatially and temporally, when compared with actual rain measurements provided by rain gauges over the same area. Specifically, we analysed three intense rain events which occurred over Israel in January 2010, January 2013 and December 2009.

This paper is organized as follows: Sects. 1.1 and 1.2 describe the different measurement sources used in this paper. Sect. 1.3 covers the study area and the details surrounding the chosen rain events. In Sect. 2 we provide a full description of the newly developed integrative approach. This is followed by results and performance evaluations of such an integrative tool, detailed in Sect. 3. Finally, conclusions and future developments of the application are provided in Sect. 4.

\subsection{The weather radar}

Over a period spanning more than half a century, starting in the late 1940s, radars have been used for estimating rainfall measurements, as initially proposed by Marshall et al. (1947). Among these radars, short-range weather radars have also been a subject of increasing interest by many researchers, even to this day (Charvat et al., 2014). The wellknown empirical relation between the radar reflectivity and the rainfall intensity is shown in Eq. (1):

$Z=a r^{b}$,

where $r$ is the rain rate (in $\mathrm{mm} \mathrm{h}^{-1}$ ), $Z$ is the radar reflectivity (in $\mathrm{mm}^{6} \mathrm{~m}^{-3}$ ), and $a$ and $b$ are known constants, which are mainly a function of the drop size distribution (DSD). These parameters may vary according to different rain types both between and within storms, which can lead to high levels of error and uncertainty in the radar, as discussed in Morin et al. (2003). Additional sources of the radar uncertainties (Germann et al., 2006) include attenuation at $\mathrm{C}$ and $\mathrm{X}$ bands, bright band contamination, and clutter regions.

Additionally, the spatial expansion effect of the radar beam results in an increase in the reflective volume by up to a few kilometres, which may lead to partial beam filling; this in turn may result in over (or under) estimation of the rain rates. Moreover, radar measurements aloft are uncertain estimates of near-ground rainfall due to ground clutter and changes in
DSD as a function of height due to evaporation, coalescence, raindrop collection and breakup (Prat and Barros, 2009). In order to deal with these uncertainties, different approaches have been proposed including the use of a polarimetric and Doppler weather radars (e.g. Bringi and Chandrasekar, 2001; Doviak et al., 1979). Such systems use the shape of the rain drops and allow for improvement in rain rate estimation (Meischner et al., 1991).

Another limiting factor in the radar accuracy is the location of the radar. The received signal can provide reasonable rainfall estimation for up to around $100 \mathrm{~km}$, though this is also dependent upon topography and the height of the radar beam. Thus, for objects too close to the radar (i.e. $<1 \mathrm{~km}$ ), or too far (i.e. $>100 \mathrm{~km}$ ), the reconstruction is characterized by much uncertainty. In cases where the distance is greater than $150 \mathrm{~km}$, the radar cannot provide an estimation. In other words, the weather radar inaccuracy increases as the distance to the target area grows (with respect to $R^{2}$ ). This fact can also be derived from the radar equation (Skolnik, 1962)

$P_{\mathrm{r}}=P_{\mathrm{t}} \tau G^{2} \lambda^{2} \theta^{2} \frac{c}{512 \pi^{2}} \frac{\eta}{R^{2}} \propto \frac{\text { Const }}{R^{2}}$,

where $P_{\mathrm{r}}$ is the received power, $P_{\mathrm{t}}$ is the transmitted power, $\tau$ is the temporal duration of a pulse, $G$ is the gain of the transmitting antenna, $\lambda$ indicates the radar wavelength, $\theta$ is the beam width (in radians), $c$ is the speed of light, and $\eta$ (in $\mathrm{dB} \mathrm{km}$ ) is the radar cross section (RCS) of the target area (Mackenzie et al., 1993). $R$ is the distance from the transmitter to the target area. One can see that the more distant the area (target) is from the radar, the lower the received signal (denoted as $P_{\mathrm{r}}$ ) is, and hence the increase in inaccuracy.

\subsection{Microwave links - ML}

A wireless microwave signal's strength, also known as RSL (received signal level), is greatly affected by precipitation (mainly rain). The well-known empirical attenuation-rainrate relation is given by (Olsen, 1978)

$A=\alpha R^{\beta} L$,

where $A$ (expressed in $\mathrm{dB}$ ) is the measured RSL; $R$ (expressed in $\mathrm{mm} \mathrm{h}^{-1}$ ) is the path averaged rain rate (along the microwave link); $L$ (expressed in $\mathrm{km}$ ) is the link length; and $\alpha$ and $\beta$ are constants, depending mainly on the link frequency and DSD, as discussed in Örs et al. (1999).

The RSL is measured by a variety of antennas distributed in space (e.g. Fig. 1), with typical frequencies of $18-23 \mathrm{GHz}$, and lengths that vary by between 1 and $20 \mathrm{~km}$. The measurements are given in a preset temporal resolution, with a known quantization level. Because the goal is to reconstruct accurate rain field maps, we inspect only significant rain events; thus, we may assume that the RSL has very high signal-tonoise ratio (SNR). A typical such RSL is shown in Fig. 2. In the figure, the RSL is provided with a magnitude resolution of $0.1 \mathrm{~dB}$, at $15 \mathrm{~min}$ sampling rate, for a link located in 

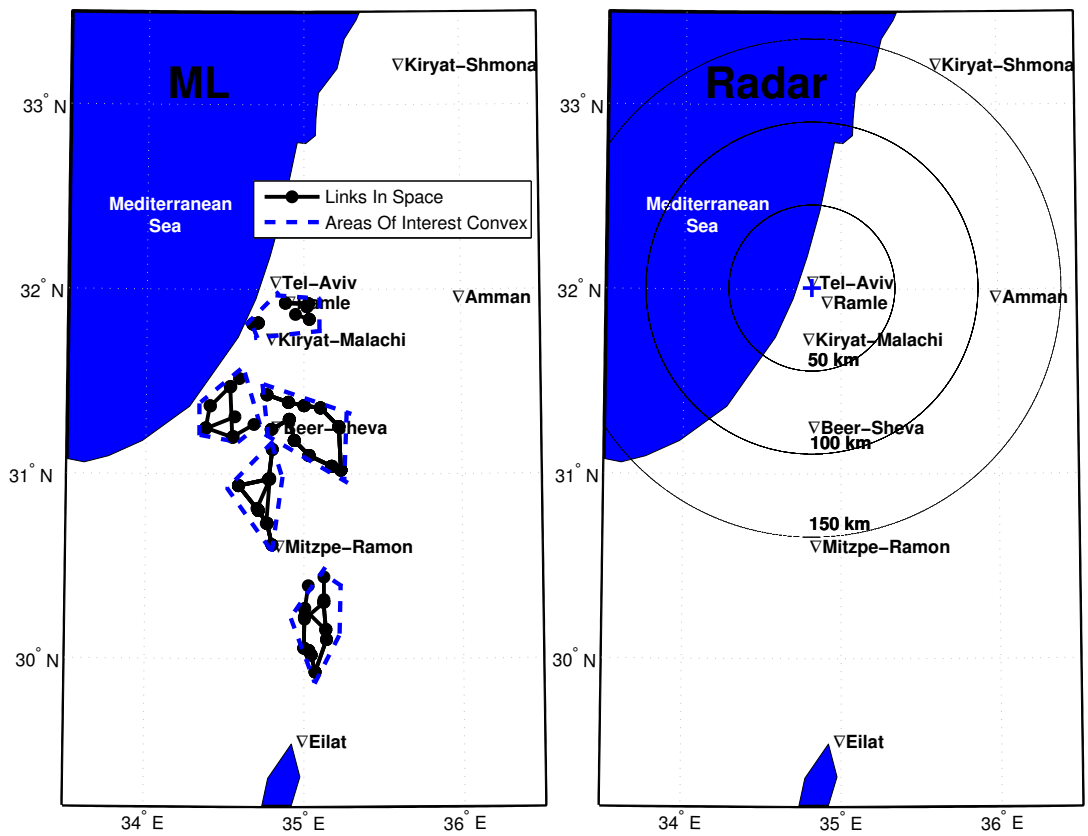

Figure 1. Left: $96 \mathrm{ML}$ in space divided into 6 areas of interest. Black lines indicate the links, and the blue dashed lines indicate the convex of each area of interest. Right: the radar coverage area, where the blue “+” sign indicates the radar coordinates - Bet Dagan. The radar's 50, 100 and $150 \mathrm{~km}$ radius distances are also indicated.

the centre of Israel (between Ramle and Hasmonaim; provided by Cellcom Ltd.). In order to overcome non-linearities in the measurements, the RSL is presented after a preprocessing stage, as detailed in the M.Sc. thesis of Liberman (2013).

Since the use of commercial ML for rainfall monitoring was first suggested by Messer et al. (2006), multiple methodologies for rainfall estimation and mapping have been suggested (e.g. Goldshtein et al., 2009; Overeem et al., 2013). Furthermore, the use of ML for precipitation monitoring in general and rainfall monitoring in particular has been a subject of interest for many researchers all around the world since 2006, and remains so to this day (e.g. Chwala et al., 2012; Overeem et al., 2014, and references therein).

In this paper, a novel algorithm which has been recently developed for recovering instantaneous rainfall maps using RSL measurements is used in the proposed integration algorithm. The basis of this algorithm is described as follows, and more details can be found in Liberman and Messer (2014).

For any given set of RSL measurements from ML, the goal is to construct the most accurate approximation of the rain rate along the links, and then to reconstruct the rain field in the link's vicinity. Suppose we have a set of observed rainfall-induced RSL attenuations from $M$ ML in a given geometry (denoted as $A_{j}$, for $j=1, \ldots, M$ ). By modifying Eq. (3), each link's RSL can be written as

$A_{j}=\alpha_{j} R_{j}^{\beta_{j}} L_{j}=\int_{L_{j}} \alpha_{j} r^{\beta_{j}}(x) \mathrm{d} x$, where $r(x)$ (expressed in $\mathrm{mm} \mathrm{h}^{-1}$ ) is the true instantaneous rain rate at a point $x$ along the link, $L_{j}$ (expressed in $\mathrm{km}$ ) is the $j$ th link length, and $\alpha_{j}$ and $\beta_{j}$ are the known $j$ th link constant parameters (Örs et al., 1999). Now, by dividing each link into $n_{j}$ (small enough) equal segments, we may approximate the integral in Eq. (4) and derive the following nonlinear relation between each link's RSL and the actual rain rate along it (i.e. along an arbitrary line in space):

$A_{j} \approx \alpha_{j} \sum_{i=1}^{n_{j}} r_{i j}^{\beta_{j}} l_{i j}$

where $l_{i j}\left(l_{i j} \ll L_{j}\right)$ is the length of the $i$ th segment for each $j$ th link and $r_{i j}$ is the unknown rain rate in each $l_{i j}$ segment.

In order to solve Eq. (5), the algorithm uses the fact that the rain field is generally represented in a sparse manner, as has often been observed in the literature (e.g. Morin et al., 2006). This means that, for some extent of the rain field, it is reasonable to assume that the solution for each $r_{i j}$ (denoted as the rain rate for each $j$ th link in each $i$ th segment where the ML are available) is mostly sparse. Therefore, the optimization $L_{1}$ problem (as discussed in Chen et al., 1998) can be solved. By doing so, a unique and optimal recovery of the rain rates along the ML can be guaranteed if some regularity conditions, mainly regarding the links distribution and the derived solution for the $L_{1}$ problem, are satisfied.

The next step is to construct a 2-D rain field map from the estimated solution of Eq. (5). That can be achieved by using either parametric or non-parametric interpolation methods. 


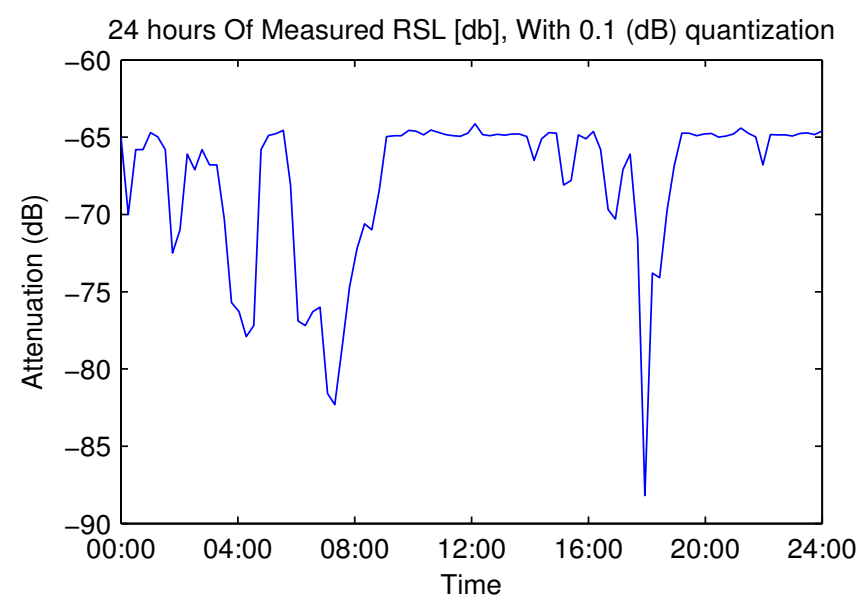

Figure 2. Example of $24 \mathrm{~h}$ of measured RSL (dB), during a rain event which occurred on 7 January 2013 , for a single $14 \mathrm{~km}$ link operating at a frequency of $21.8 \mathrm{GHz}$.

\subsection{Available data and coverage area}

The coverage area of the radar includes all the coordinates in space that lie within $0.1 \leq R_{i} \leq 150 \mathrm{~km}$ from the radar location, where $R_{i}$ indicates the radar radius distance from each $\left[x_{i}, y_{i}\right]$ coordinate. In this study, the data from the weather radar, which is located in Bet Dagan $\left(32.007^{\circ} \mathrm{N}, 34.814^{\circ} \mathrm{E}\right)$, is provided by the Israel Meteorological Service (IMS - see Fig. 1). The measurements from the radar are provided at a resolution of $1 \mathrm{~km}^{2}$ every $5 \mathrm{~min}$. Though the radar employed in Israel has "automatic clutter removal" (Skolnik, 1962), dominant clutters can still be observed, mainly in the north of Israel, where many hilly areas are found (e.g. in Ramat HaGolan: $32.58^{\circ} \mathrm{N}, 35.44^{\circ} \mathrm{E}$ ).

Regarding the ML, we define the covered areas dependent upon the location of the specific ML. Here, operational ML data in central-southern Israel was provided by Cellcom Ltd. (i.e. 96 operating microwave links) and Pelephone Ltd. (30 operating microwave links), as shown in Fig. 1. The ML operate at frequencies of $18-23 \mathrm{GHz}$ and are horizontally or vertically polarized, with lengths that range from 3 to $20 \mathrm{~km}$ and with magnitude resolutions of $0.1 \mathrm{~dB}$ for Cellcom Ltd. and $1 \mathrm{~dB}$ for Pelephone Ltd., which may cause a degradation in the accuracy of the estimated rainfall using ML. Still, because we analysed only heavy rain events in this study (highSNR data, Sect. 1.2), the effect of the magnitude resolution on the reconstruction accuracy is negligible. In addition, 15 min time intervals of minimum and maximum RSL values were provided by Cellcom and $1 \mathrm{~min}$ of temporal resolution data were provided by Pelephone Ltd. In this study, three major rain events were chosen for the analysis: event 1,18 19 January 2010 (24h of rain); event 2, 7-10 January 2013 (96h of rain); and event 3, 30 December 2009 ( $24 \mathrm{~h}$ of rain).

Rainfall estimates from the set of events 1, 2 and 3 were validated against a network of 70 tipping-bucket rain gauges

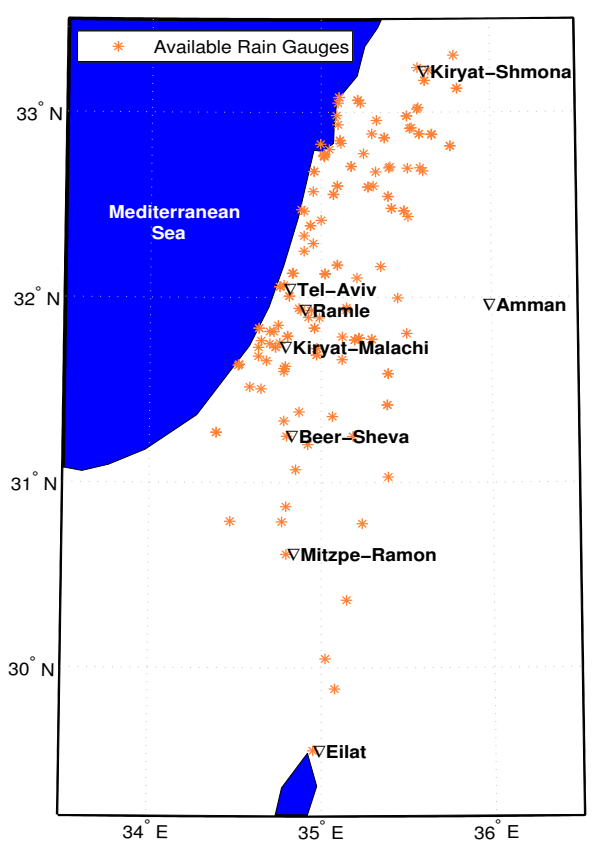

Figure 3. Distribution of the 70 available rain gauges; each orange asterisk indicates a rain gauge location.

distributed throughout the region (as illustrated in Fig. 3) recording at a time resolution of $10 \mathrm{~min}$, where each rain gauge provides ground truth (accumulated) rain measurements in millimetres, i.e. water volume per $\mathrm{m}^{2}$.

\section{The integrative approach}

In this section, we detail the stages of the integration technique, whose main goal is to combine the different rainfall measurements in a way which optimally weights the pros and cons of the various methods. As mentioned above, the radar data are provided by the IMS, while the RSL data are provided by the cellular companies Cellcom and Pelephone.

Data assimilation is widely used in many environmental fields, particularly in weather modelling (e.g. Chahine et al., 2006) and in hydrological modelling (e.g. McLaughlin, 2002). In data assimilation, we are required to form a relationship between the state we want to estimate (e.g. the 2-D distribution of rainfall intensity) and the different observation sources (e.g. radar and ML). Thus, we can assume that the process relationship between the observations and the desired estimate is represented by a forward model (denoted as $f$ ), which is defined in Eq. (6):

$R_{\mathrm{int}}\left(x_{i}, y_{i}\right)=f\left(R_{\mathrm{rad} ; i}, R_{\mathrm{ml} ; i}\right)$,

where $R_{\text {int }}\left(x_{i}, y_{i}\right)$ is the required state (rainfall intensity, in each $x_{i}, y_{i}$ coordinate in space), and $R_{\mathrm{rad} ; i}$ and $R_{\mathrm{ml} ; i}$ are the rainfall intensities obtained by the radar and the ML observations, respectively, for each $x_{i}, y_{i}$ coordinate in space (where 
data are available). Figure 1 shows the study area where data from radar and/or ML are available (i.e. in the case of the ML, we divided the space into areas of interest).

In order to generate the most reliable reconstruction of rainfall maps, we define the following rules, based on the characteristics of the different measurement sources:

1. For all the coordinates which are not covered by ML (i.e. the coordinates where the reconstruction by the ML is not available, e.g. in Fig. 1), we only regard the reconstruction received by the radar, if available, i.e. any $\left[x_{i}, y_{i}\right]$ coordinate which is not covered by the ML but satisfies $0.1 \leq R_{i} \leq 150$.

2. Inside an area of interest, with available ML, we check whether both the following regularity conditions are satisfied:

a. The distribution of the links in space satisfy the reconstruction ability condition, as discussed and proven in Sendik and Messer (2012), which states that by having enough ML in some area of interest, it is possible to achieve a highly reliable recovery of the rainfall along the microwave links in that area.

b. The estimated rain intensities in space along the microwave links (which are distributed in an arbitrary manner in space) satisfy the generalized ShannonNyquist sampling theorem for non-uniform sampling, as detailed in Eldar (2003).

3. If condition 1 and condition 2 are not satisfied inside an area of interest, we apply a new weighted algorithm using both of the sources in the study area. The rainfall measurement will then rely both on the links distribution in space and the radar radius, as shown in Eq. (2).

If condition 2 is satisfied in a given area, an optimal recovery of the rainfall, using ML, in that area is possible (see Sect. 1.2). Hence, we do not consider the radar reconstruction in that area at all. If condition 3 is satisfied, we use some sort of integration scheme between the ML and the radar. For this integration we propose a weighted linear model, mainly due to the fact that linear models have been adopted and have proven useful (e.g. Daley, 1993) in previous data assimilation works, especially for hydrological and weather forecasts.

It should be noted that both the radar and the ML data undergo some preprocessing stage before the integration is applied. For example, dominant clutter areas (denoted as Clutter), which are characterized by much uncertainty in the radar reconstruction (see Sect. 1.3), are determined by using prior information and rain gauge measurements. Regarding the ML RSL data, a zero-level reduction, noise removal and other preprocessing methods are applied before the rainfall maps are created, as further discussed in Liberman (2013). Hence, by using the proposed conditions above, we may rewrite $R_{\text {int }}$ in Eq. (6) as follows Our:

$R_{\mathrm{int}}\left(x_{i}, y_{i}\right)=\left\{\begin{array}{l}R_{\mathrm{rad} ; i}, 0.1 \mathrm{~km} \leq R_{i} \leq 150 \mathrm{~km} \cap N_{i}=0 \\ R_{\mathrm{ml} ; i}, N_{i}>1 \cap\left(R_{i} \geq 150 \mathrm{~km} \cup R_{i}\right. \\ \leq 0.1 \mathrm{~km} \cup \text { Condition } 2 \cup \text { Clutter }) \\ f_{\mathrm{Lin}}\left(R_{\mathrm{ml} ; i}, R_{\mathrm{rad} ; i}\right), N_{i}>0 \cap 0.1 \mathrm{~km} \\ \leq R_{i} \leq 150 \mathrm{~km} \cap \text { No Clutter }\end{array}\right.$

In Eq. (7), $R_{\mathrm{rad} ; i}$ and $R_{\mathrm{ml} ; i}$ are the rain rate values, in the $\left[x_{i}, y_{i}\right]$ coordinates, for the radar and ML, respectively. $N_{i}$ indicates the number of links in the area of interest that the $\left[x_{i}, y_{i}\right]$ coordinate belongs to. $R_{i}$ is the distance (denoted as the radar radius, expressed in $\mathrm{km}$ ) between the radar location and each $\left[x_{i}, y_{i}\right]$ coordinate in space. $\cup$ and $\cap$ indicate the OR and AND operators, respectively. $f_{\text {Lin }}$ is a linear function of $R_{\mathrm{ml} ; i}$ and $R_{\mathrm{rad} ; i}$, which is defined by

$f_{\mathrm{Lin}}\left(R_{\mathrm{ml} ; i}, R_{\mathrm{rad} ; i}\right)=\tilde{\alpha}_{\mathrm{rad} ; i} R_{\mathrm{rad} ; i}+\tilde{\alpha}_{\mathrm{ml} ; i} R_{\mathrm{ml} ; i}$,

where $f_{\text {Lin }}$ is calculated in each $\left[x_{i}, y_{i}\right]$ "common" coordinate (i.e. where rain intensities are provided both by the radar and ML) in space. $\tilde{\alpha}_{\mathrm{rad} ; i}$ and $\tilde{\alpha}_{\mathrm{ml} ; i}$ denote the normalized radar and ML weights, respectively. These weights are a function of the radar radius (denoted as $R_{i}$ ) and the number of links in the area of interest (denoted as $N_{i}$ ) in each $\left[x_{i}, y_{i}\right]$ common coordinate (i.e. where condition 3 is satisfied). Since $\tilde{\alpha}_{\mathrm{rad} ; i}$ and $\tilde{\alpha}_{\mathrm{ml} ; i}$ are subject to $\tilde{\alpha}_{\mathrm{rad}}+\tilde{\alpha}_{\mathrm{ml}}=1$, we may model these weights as follows:

$\tilde{\alpha}_{\mathrm{rad} ; i}=\frac{\alpha_{\mathrm{rad}}}{\alpha_{\mathrm{rad}}+\alpha_{\mathrm{ml}}}$

$\tilde{\alpha}_{\mathrm{ml} ; i}=\frac{\alpha_{\mathrm{ml}}}{\alpha_{\mathrm{rad}}+\alpha_{\mathrm{ml}}}$,

$\alpha_{\mathrm{rad}}$ and $\alpha_{\mathrm{ml}}$ are denoted as the radar and ML nonnormalized weights, respectively. From Eq. (9), it is clear that $\tilde{\alpha}_{\mathrm{ml}}+\tilde{\alpha}_{\text {rad }}=1$; hence, this offered model is valid.

As mentioned before, the accuracy in the reconstruction of rain fields, derived by the radar and the ML, is mainly dependent on the number of links and the radar radius (distance from the target area) in each coordinate. Thus, for each $\left[x_{i}, y_{i}\right]$ coordinate which satisfies condition 3 , we can model $\alpha_{\mathrm{ml}}$ and $\alpha_{\text {rad }}$ as follows:

$\alpha_{\mathrm{rad}}=\frac{c_{\mathrm{r}}^{2}}{c_{\mathrm{r}}^{2}+R_{i}^{2}}$

$\alpha_{\mathrm{ml}}=\frac{N_{i}^{2}}{N_{i}^{2}+c_{1}^{2}}$,

where $c_{\mathrm{r}}$ and $c_{1}$ are the radar and ML weight constants, respectively. From the definition of the weights in Eq. (10), it is clear that, as $N_{i}$ is higher, $\tilde{\alpha}_{\mathrm{ml} ; i}$ is higher and, accordingly, as $R_{i}$ is lower (closer to the radar location), $\tilde{\alpha}_{\text {rad; } i}$ is higher; thus, 
it is the natural choice of the weights. It should be noted that other forms of weights (e.g. exponential weights) were also considered for the integration, but these have been proven to be less accurate than the ones proposed here. Future work may focus on other forms of weights for the integration. Now, by substituting Eq. (10a) into Eq. (9a), and Eq. (10b) into Eq. (9b), we may derive the following relation:

$\tilde{\alpha}_{\mathrm{rad} ; i}=\frac{\left(N_{i}^{2}+c_{1}^{2}\right) c_{\mathrm{r}}^{2}}{\left(N_{i}^{2}+c_{1}^{2}\right) c_{\mathrm{r}}^{2}+\left(c_{\mathrm{r}}^{2}+R_{i}^{2}\right) N_{i}^{2}}=h_{i}\left(c_{1}, c_{\mathrm{r}}\right)$,

$\tilde{\alpha}_{\mathrm{ml} ; i}=\frac{\left(c_{\mathrm{r}}^{2}+R_{i}^{2}\right) N_{i}^{2}}{\left(N_{i}^{2}+c_{1}^{2}\right) c_{\mathrm{r}}^{2}+\left(c_{\mathrm{r}}^{2}+R_{i}^{2}\right) N_{i}^{2}}=1-h_{i}\left(c_{1}, c_{\mathrm{r}}\right)$.

The variable $h_{i}\left(c_{1}, c_{\mathrm{r}}\right)$ is a non-linear function of the unknown scalar variables $-c_{1}, c_{\mathrm{r}}$, while for each $\left[x_{i}, y_{i}\right]$ coordinate, $N_{i}$ and $R_{i}$ are known. Now, if we substitute Eq. (11) into $f_{\mathrm{Lin}}\left(R_{\mathrm{ml} ; i}, R_{\mathrm{rad} ; i}\right)$ in Eq. (7), we derive the following relation between $f_{\mathrm{Lin}}\left(R_{\mathrm{ml} ; i}, R_{\mathrm{rad} ; i}\right)$ and $h_{i}\left(c_{1}, c_{\mathrm{r}}\right) \triangleq h_{i}$, that is:

$f_{\mathrm{Lin}}\left(R_{\mathrm{ml} ; i}, R_{\mathrm{rad} ; i}\right)=h_{i} R_{\mathrm{rad} ; i}+\left(1-h_{i}\right) R_{\mathrm{ml} ; i}$,

where one can see that $0 \leq h_{i} \leq 1$.

Now, by defining $R_{\mathrm{g} ; i}$ as the actual rain intensity at each $\left[x_{i}, y_{i}\right]$ coordinate, as measured by the rain gauges, we can minimize the cost function, as defined in Eq. (13), in order to derive the optimal solution for the unknown variables (i.e. for $\left.\left[c_{1}, c_{\mathrm{r}}\right]\right)$ :

$C\left(c_{1}, c_{\mathrm{r}}\right)=\sum_{i}\left(h_{i} R_{\mathrm{rad} ; i}+\left(1-h_{i}\right) R_{\mathrm{ml} ; i}-R_{\mathrm{g} ; i}\right)^{2}$.

By minimizing $C\left(c_{1}, c_{\mathrm{r}}\right)$ in Eq. (13), we may derive our estimate for $\left[c_{1}, c_{\mathrm{r}}\right]$, which is given by

$\left[\hat{c}_{1}, \hat{c}_{\mathrm{r}}\right]=\underset{c_{1}, c_{\mathrm{r}}}{\operatorname{argmin}}\left\{C\left(c_{1}, c_{\mathrm{r}}\right)\right\}$.

We point out that each $R_{\mathrm{g} ; i}$ (denoted as the rain gauge in the $\left[x_{i}, y_{i}\right]$ coordinate) provides measurements of the amount of rain (in $\mathrm{mm}$ ) for a certain amount of time. Therefore, in order to derive the desired estimates, as shown in Eq. (14), we analysed three heavy rain events which have occurred over the last 5 years in Israel, specifically on 18 January 2010, 7-10 January 2013 and 30 December 2009. We obtained an estimate for the unknown variables $\left[c_{1}, c_{\mathrm{r}}\right]$ using the rain intensities available from the radar, ML, and rain gauges for all available coordinates where each inspected coordinate satisfied condition 3 from above and a rain gauge measurement was available at that coordinate as well. The non-linear estimation problem in Eq. (14) might be solved in various ways (e.g. Marquardt, 1963; Wan and Van Der Merwe, 2000). In this research we used a non-linear least-squares iterative method, as discussed in Byrd et al. (1987).

\section{Results}

This section describes the results of the rainfall measurements from different sources (only radar, only ML, and the integrated method). The first two parts define the study area and parameter estimation, respectively. In the third part we present the results, including reconstructed maps, scatter plots, performance evaluations, and graphs showing statistical and numerical comparisons.

\subsection{Study area}

The study area is located in the centre of Israel (approximately $22000 \mathrm{~km}^{2}$ ), where both radar and ML data are available. Most of the region (from the north to the centre) is covered by the IMS radar, located in Bet Dagan, as shown in Fig. 1, where the areas covered by ML are also delineated.

The rain rates for calibration and validation of the rainfall measurements were recorded by 70 rain gauges distributed in space (see Fig. 3). Moreover, the total attenuation of 96 operational telecommunication ML was also used. The ML operate at a time resolution of $15 \mathrm{~min}$; the radar operates at a spatial resolution of $1 \mathrm{~km}^{2}$ with $5 \mathrm{~min}$ time intervals; and the rain gauge network, composed of 70 tipping-bucket gauges, provides measurements at a time resolution of $10 \mathrm{~min}$.

In order to make the data from the rain gauges, ML and radar comparable, we inspect only the common times which occur every $30 \mathrm{~min}$ (i.e. at 00:00, 00:30 . . 23:30 IDT (Israel Daylight Time)). The ML used in this application operate at $18-23 \mathrm{GHz}$, with horizontal (or vertical) polarization, with lengths that vary by between 3 and $20 \mathrm{~km}$ and with a magnitude resolution of $0.1 \mathrm{~dB}$. The reconstruction adopted for the ML is the instantaneous rain field reconstruction developed by Liberman et al. (2014) as described in Sect. 1.2; however, as mentioned before, any reconstruction technique can be applied (e.g. Zinevich et al., 2010; Overeem et al., 2013) for the proposed analysis as well.

\subsection{Parameter estimation}

For the estimation of the parameters, we used 40 different points in space, all of which had available data from all sources. Given the above, we have chosen two of the three events mentioned above for the provided analysis of the new technique by using a leave-one-out procedure for calibration and validation of the measurements. Specifically, for the set of rain events 1,2 and 3, only data from the other two events are used as follows:

1. Event 1: 7-10 January 2013, 30 December 2009;

2. Event 2: 18 January 2010, 30 December 2009;

3. Event 3: 7-10 January 2013, 18 January 2010.

Given the large amount of data, we assume that the estimations will be similar for all inspected events. The non-linear least-squares iterative unique solution yielded the following 


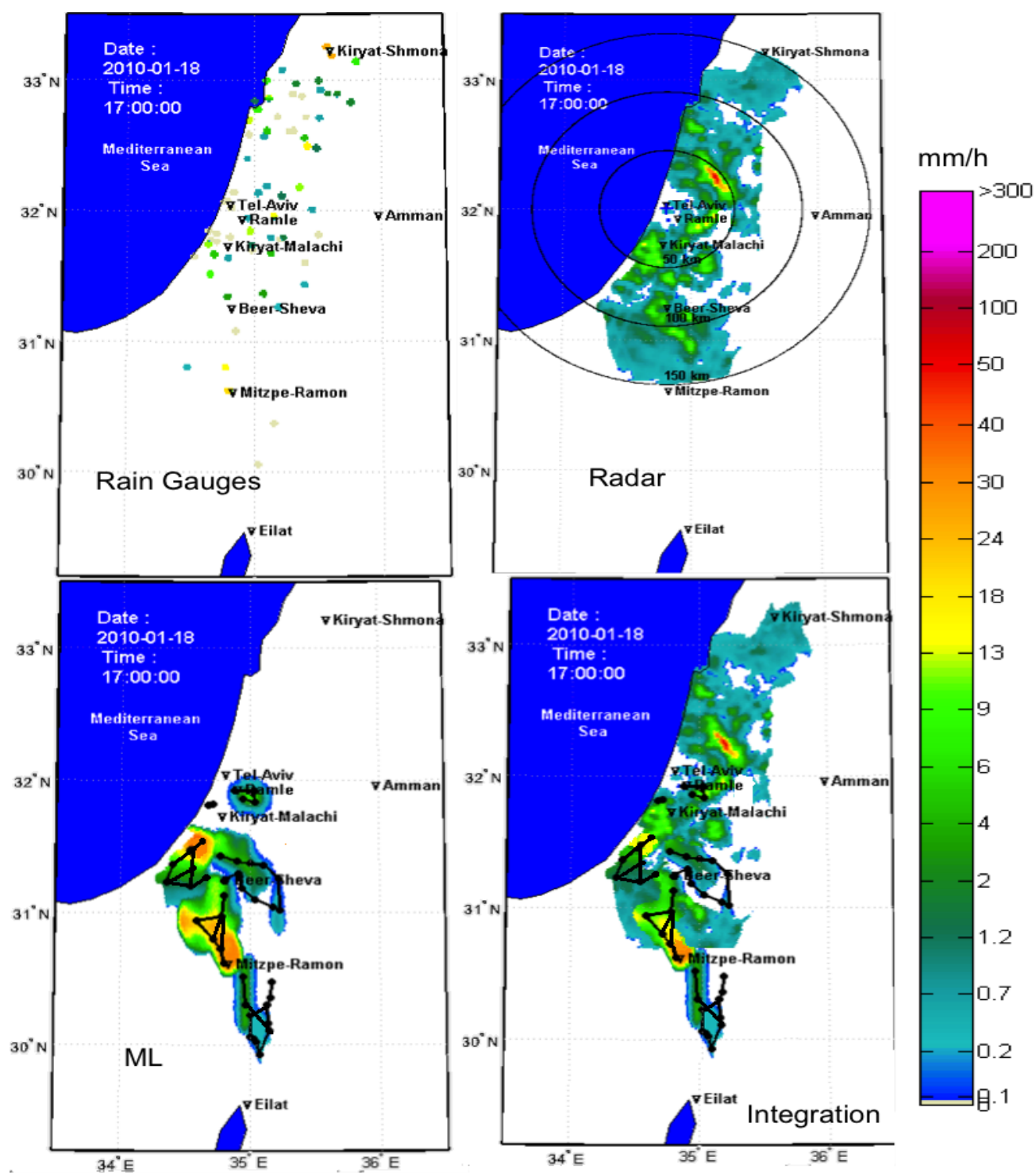

Figure 4. Example of the rain field reconstruction for the 18 January 2010 rain event (event 1) at 17:00 IDT (Israel Daylight Time). Top left: rain gauges. Top right: radar. Bottom left: ML. Bottom right: the integration.

estimation results for each one of the examined events:

$\left[\hat{c}_{1}, \hat{c}_{\mathrm{r}}\right]_{\text {Event }_{1}}=[9.82,98.89]$,

$\left[\hat{c}_{1}, \hat{c}_{\mathrm{r}}\right]_{\text {Event }_{2}}=[10.64,100.17]$,

$\left[\hat{c}_{1}, \hat{c}_{\mathrm{r}}\right]_{\mathrm{Event}_{3}}=[10.45,101.02]$.

In $(15 \mathrm{c}),\left[\hat{c}_{1}, \hat{c}_{\mathrm{r}}\right]$ are denoted as the estimations for $\left[c_{1}, c_{\mathrm{r}}\right]$ for each their respective events. While the $\hat{c}_{\mathrm{r}}$ values are similar, there is a difference of about $8 \%$ in the $\hat{c}_{1}$ estimations of event 1 with respect to events 2 and 3 . However, they are still close enough to provide reliable estimations. Moreover, as the number of measurements from rain events increases, the parameter estimation will be stronger, hence improving the application of the algorithm for future use. In short, by using these values, we assume an optimal linear weighted in- tegration when considering the radar and ML for the purpose of rain field reconstruction for each one of the inspected sets of rain events (1,2 and 3$)$.

\subsection{The reconstruction evaluation}

In order to best evaluate the performance of the different measuring techniques, we present both the rainfall maps and comparative statistics. For the purpose of comparing the different measurements to actual rain intensities over several coordinates in space, we calculate the spatial correlation, RMSE (the spatial root-mean-square error) and RB (the 


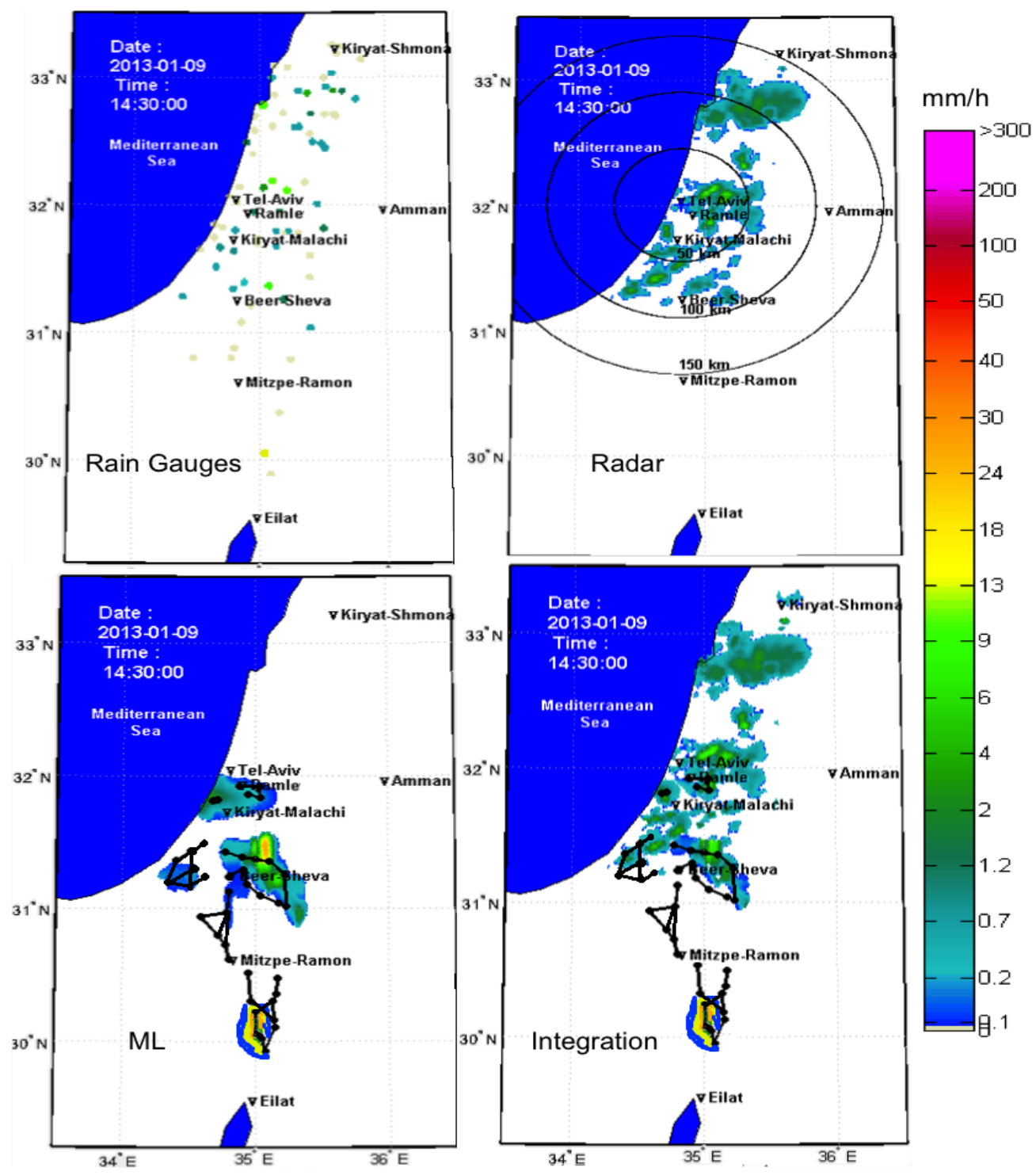

Figure 5. Example of the rain field reconstruction for the 9 January 2013 rain event (event 2) at 14:30. Top left: rain gauges. Top right: radar. Bottom left: ML. Bottom right: the integration.

relative bias, in \%), which are defined as follows:

$\rho=\frac{\sum_{j=1}^{M} \sum_{i=1}^{N}\left(\hat{x}_{i, j}-\mu_{\hat{x}}\right)\left(x_{i, j}-\mu_{x}\right)}{\sqrt{\sum_{j=1}^{M} \sum_{i=1}^{N}\left(x_{i, j}-\mu_{x}\right)^{2} \sum_{j=1}^{M} \sum_{i=1}^{N}\left(\hat{x}_{i, j}-\mu_{\hat{x}}\right)^{2}}}$,

$\operatorname{RMSE}=\sqrt{\frac{1}{N M} \sum_{j=1}^{M} \sum_{i=1}^{N}\left(\hat{x}_{i, j}-x_{i, j}\right)^{2}}$,

$\mathrm{RB}=\frac{1}{N M} \sum_{j=1}^{M} \sum_{i=1}^{N} \frac{\hat{x}_{i, j}-x_{i, j}}{x_{i, j}} \times 100$,

where $\rho$ is defined as the spatial correlation. In Eq. (16), $\mu_{\hat{x}}=\frac{1}{N M} \sum_{j=1}^{M} \sum_{i=1}^{N} \hat{x}_{i, j}$ and $\mu_{x}=\frac{1}{N M} \sum_{j=1}^{M} \sum_{i=1}^{N} x_{i, j}$ are defined as the mean spatial rain rates of the estimated rain measurements and the true measurements, respectively. In Eq. (16), the index $j$ refers to each time step (total of $M$ time steps), while index $i$ refers to each $\left[x_{i}, y_{i}\right]$ spatial coordinate in space (total of $N$ coordinates).

In Figs. 4, 5 and 6, the rain field reconstructions estimated by the different sources - rain gauges, radar, ML, and the integrated method - are illustrated. Maps are shown for a given time step for each of the analysed events (i.e. $18 \mathrm{Jan}$ uary 2010 at 17:00, 9 January 2013 at 14:30 and 30 December 2009 at 16:00 IDT). From these figures, it is clear that the integrated method expands the spatial coverage substantially.

In addition, the integrative method improved the estimations for many of the areas where radar coverage is poor and ML exist, specifically the area of Mitzpe Ramon. As can be observed, the radar cannot provide an estimate for the rain 

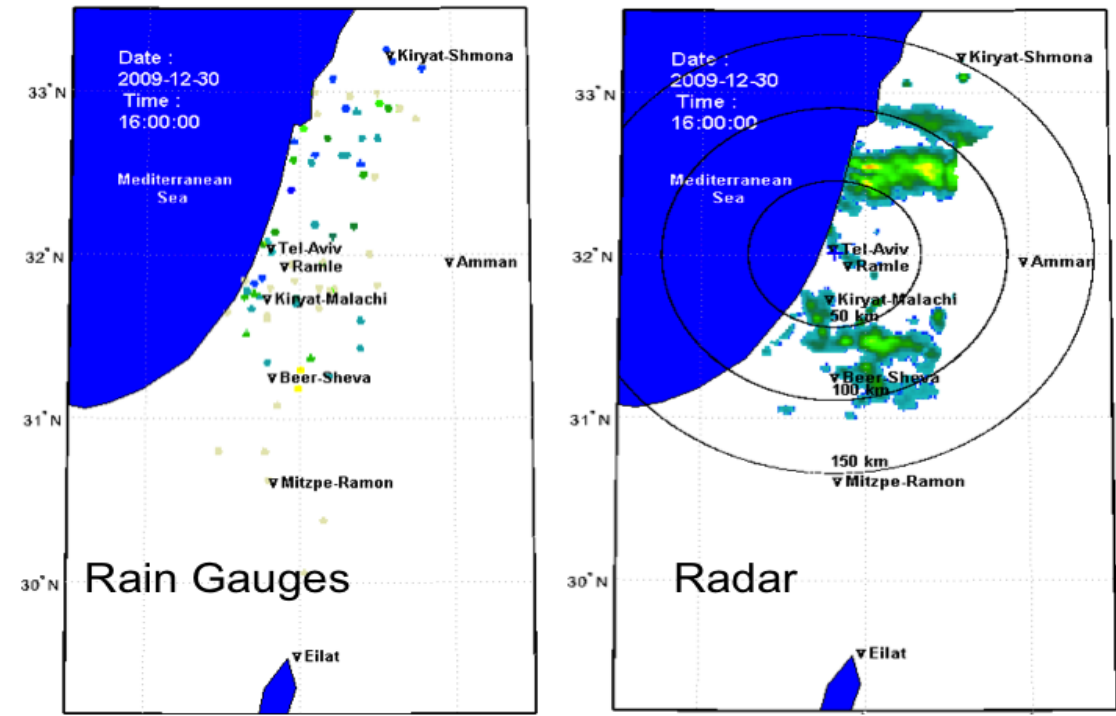

$\mathrm{mm} / \mathrm{h}$
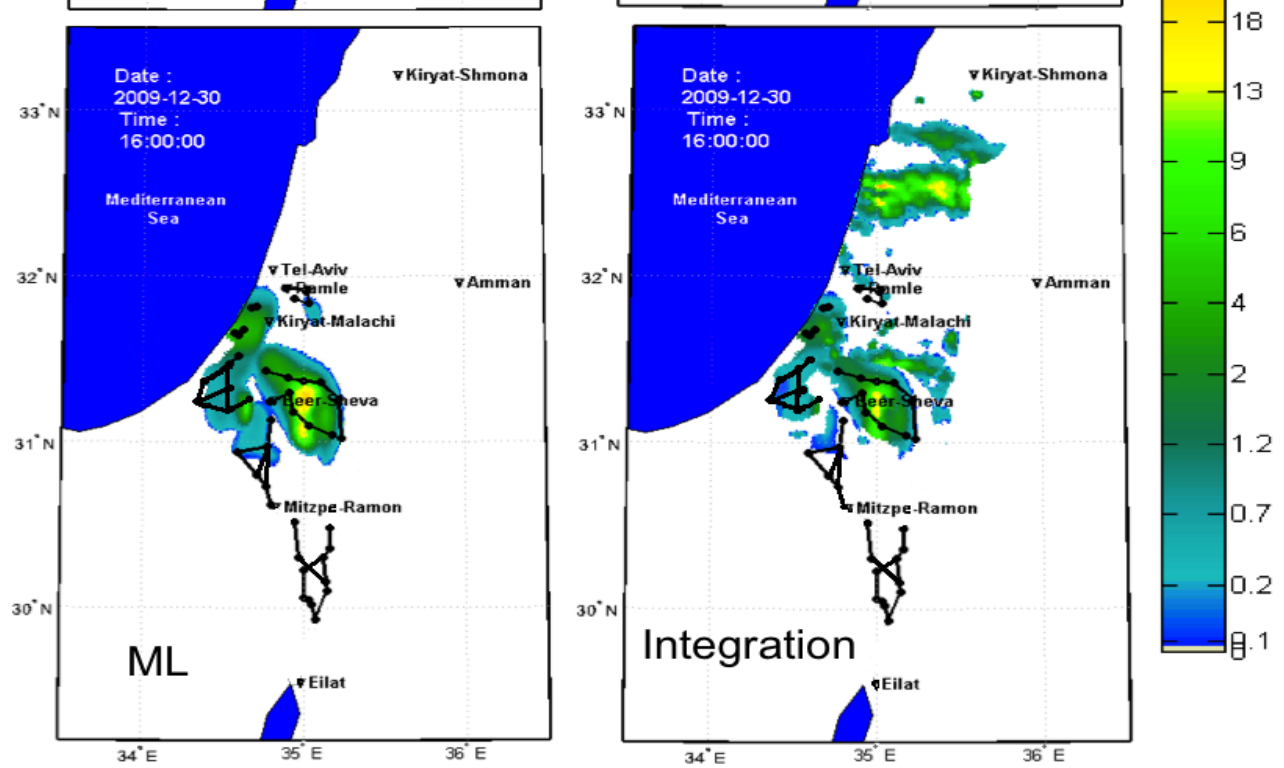

Figure 6. Example of the rain field reconstruction for the 30 December 2009 rain event (event 3) at 16:00 IDT. Top left: rain gauges. Top right: radar. Bottom left: ML. Bottom right: the integration.

rate if the distance between the radar and a coordinate in space is higher than $150 \mathrm{~km}$, as has also been discussed in Sect. 1.1.

Figures 7, 8 and 9 depict the performance evaluations for events 1,2 and 3, respectively. The correlation coefficient (spatial correlation) and the RMSE are shown, both evaluated for the common times spanning the rain event. Each line in the figures is the comparison between one of the sources as compared to actual rain intensities measured by the rain gauges. The added value of the integrated technique is evident in its lower RMSE and RB while showing higher correlation values in all events. Moreover, each method's performance was also evaluated over the entire event. This is provided in Figs. 10, 11 and 12 for events 1, 2 and 3, respec- tively, while inspecting the mean correlation, RMSE and the relative bias, as defined in Eq. (16).

Furthermore, we had also evaluated the probability of detection (POD), the false alarm ratio (FAR) and the critical success index (CSI) on the proposed integration technique and the radar. These measures are very important criteria for assessing the quality of the method. For this, we use the definition of the relative error, i.e. $\phi \triangleq \frac{|\hat{x}-x|}{x}$, where $x$ denotes a rain gauge (ground truth) measurement and $\hat{x}$ is the rain intensity estimation at the same point. Thus, a success is declared if $\phi<\epsilon$ (e.g. $\epsilon=10 \%$ ); otherwise it is regarded as a miss. A false alarm is declared if a rain gauge indicated no rain but the estimation did. Given that definition, by denoting $S$ as total successes, $M$ as total misses, and $F$ as total false 

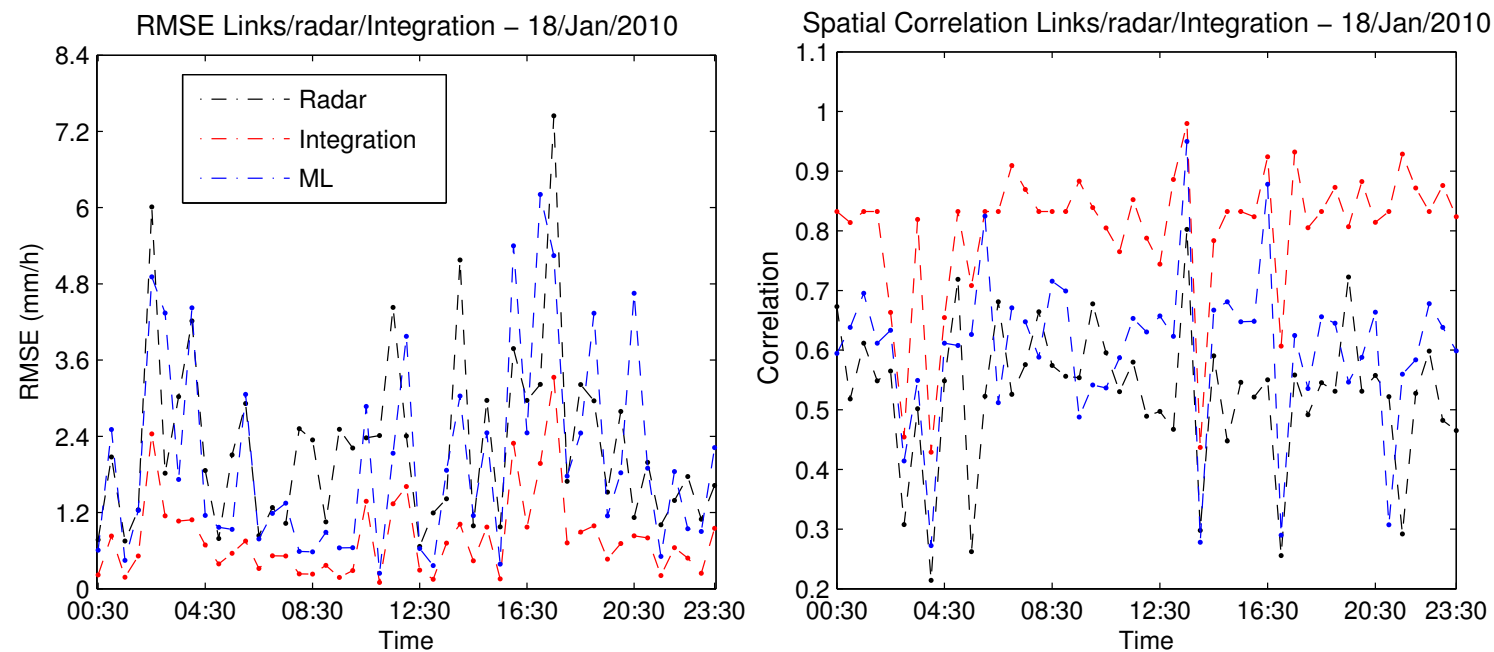

Figure 7. Evaluation analysis of event 1, where the radar (black line), the proposed integration technique (red line) and ML (blue line) are compared to the rain gauges at the common times. Left: RMSE. Right: spatial correlation.
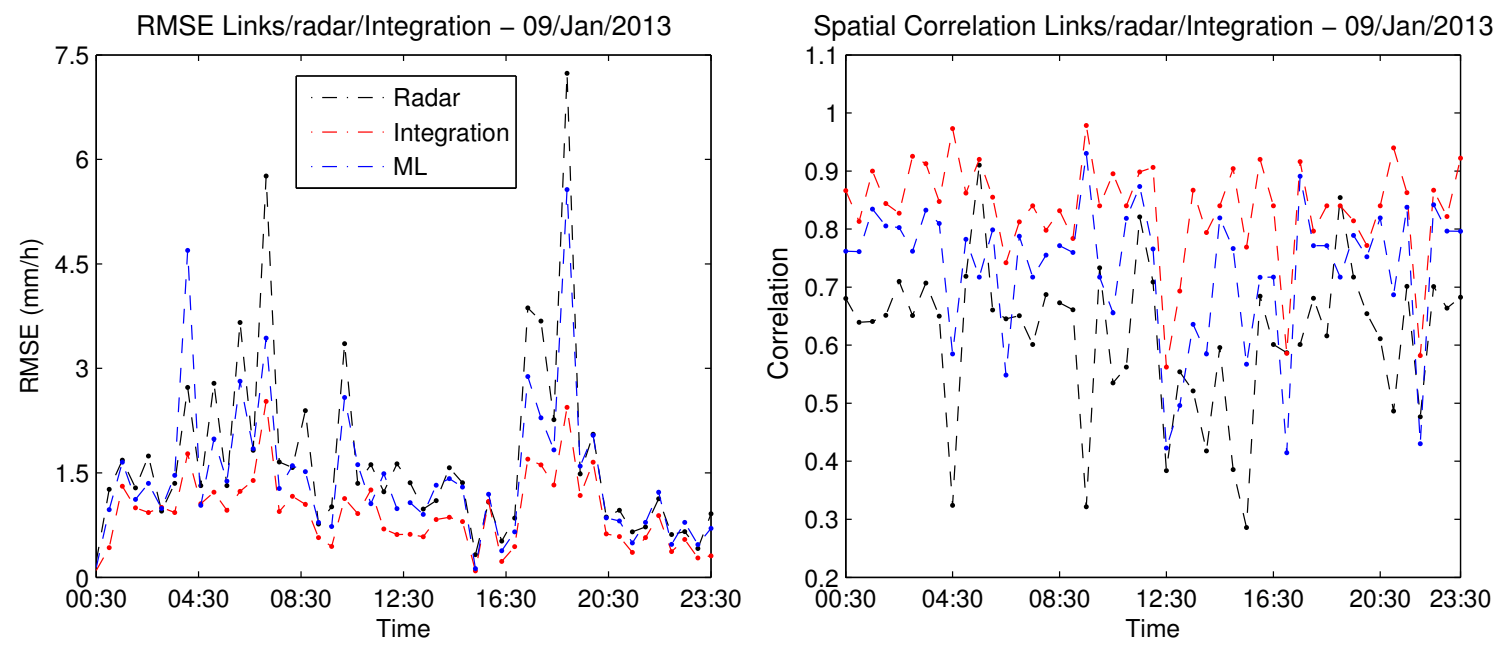

Figure 8. Evaluation analysis of event 2, where the radar (black line), the proposed integration technique (red line) and ML (blue line) are compared to the rain gauges at the common times. Left: RMSE. Right: spatial correlation.

alarms, the given criteria can be evaluated by POD $\triangleq \frac{S}{M+S}$; $\mathrm{FAR} \triangleq \frac{F}{F+S} ; \mathrm{CSI} \triangleq \frac{S}{S+M+F}$. By considering all the available rain gauge measurements, for all the inspected events at all given time frames, the integration algorithm achieved the scores of (with $\epsilon=10 \%$ ) POD $\approx 89 \%$, FAR $\approx 9 \%$, and $\mathrm{CSI} \approx 82 \%$. Under the same conditions, the radar achieved a performance of $\mathrm{POD} \approx 74 \%, \mathrm{FAR} \approx 19 \%$, and $\mathrm{CSI} \approx 68 \%$. These results once again prove the high quality of the proposed integration technique. It should be noted that, even when lower values of $\epsilon$ were used, similar results were obtained.

In addition to the performance evaluations, Figs. 10, 11 and 12 also demonstrate the scatter plots, each with its corresponding regression line (black line) for events 1, 2 and 3, respectively.
From these figures it is clear that the disparity of the points is the lowest for the integration algorithm with respect to the ML and the radar scatter plots (due to the under and over estimation of their reconstructions). This implies that the integrative approach is the most accurate one.

The highest correlations, lowest RMSE and lowest (absolute) RB for all three rain events were obtained using the integration algorithm. From both the maps and the comparative statistics, the integrated method provides a new way to improve rainfall estimation spatially and over time. The effectiveness of using this particular integration scheme, as in Eq. (7), can be understood by examining the RB of the radar and ML, which showed an over- and underestimation, respectively, for all the inspected events. 

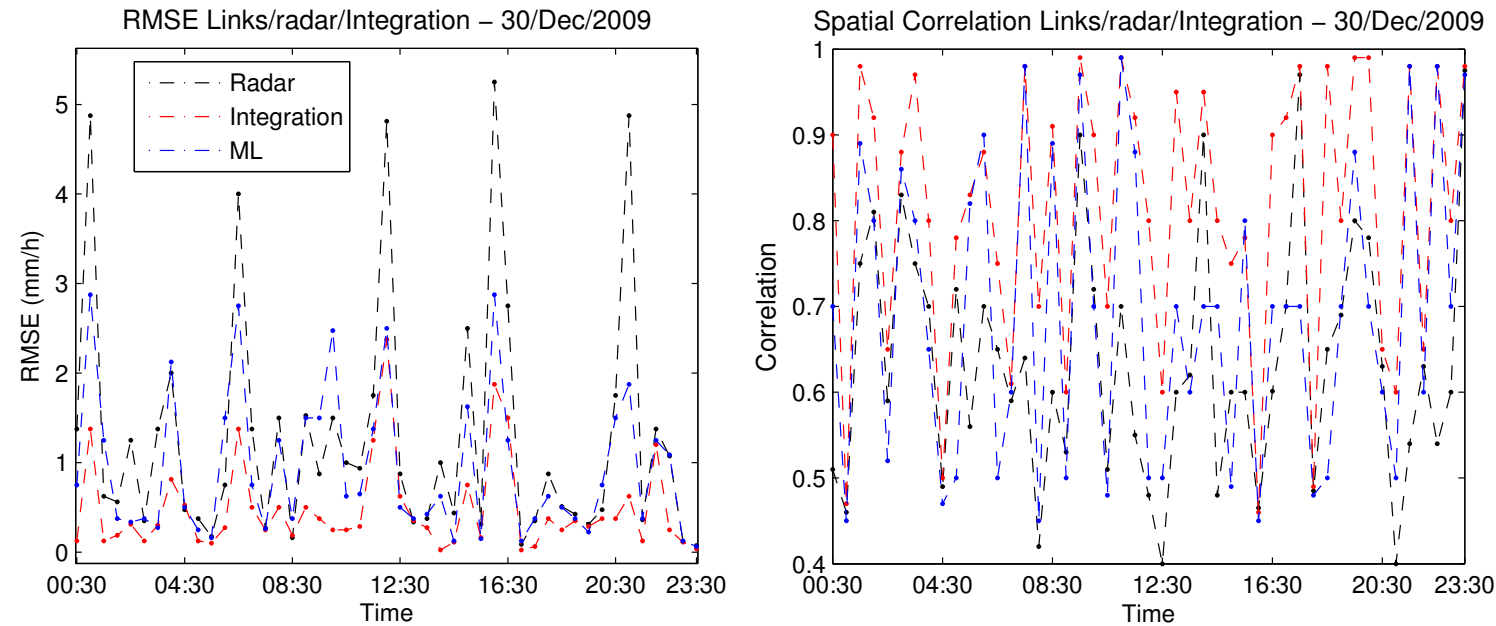

Figure 9. Evaluation analysis of event 3, where the radar (black line), the proposed integration technique (red line) and ML (blue line) are compared to the rain gauges at the common times. Left: RMSE. Right: spatial correlation.
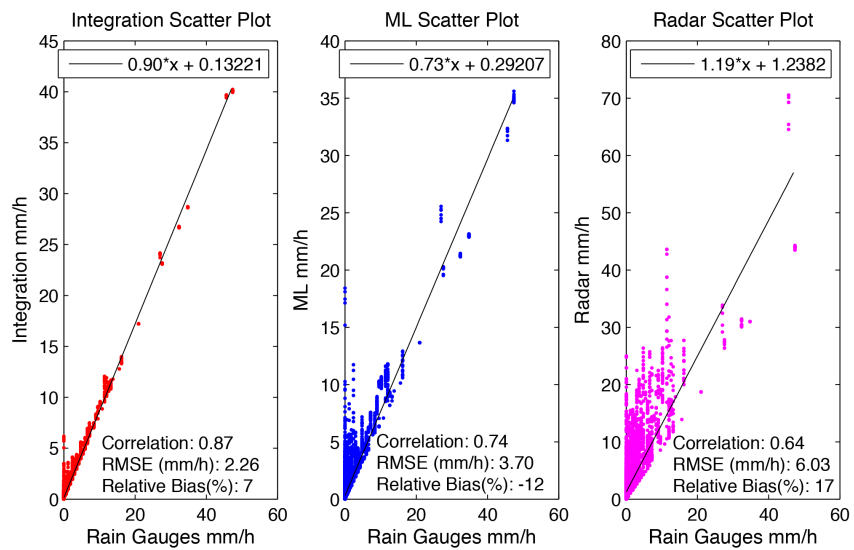

Figure 10. Rain rate scatter plots and performance evaluations of the integration (left), ML (middle) and radar (right) with respect to all the available rain gauges for event 1 .

Our last analysis of the data was comparing the total accumulated rainfall over a specific point located inside the study area for the duration of the rain event. The accumulated rain was calculated as defined in the following relation:

$R\left(T_{k}\right)=\int_{0}^{T_{k}} r_{i j} \mathrm{~d} t \approx \sum_{r=0}^{T_{k}} r_{i j}\left(T_{\mathrm{r}}\right) \Delta T(\mathrm{~mm})$,

where $r_{i j}$ is the rain rate $\left(\mathrm{mm} \mathrm{h}^{-1}\right)$ in the $\left[x_{i}, y_{j}\right]$ coordinate, $\Delta T$ is the time resolution (e.g. for the rain gauges $1 / 6 \mathrm{~h}$ ), $T_{k}$ is the accumulation time, $T_{\mathrm{r}}$ indicates each time sample for each $r_{i j}$ (i.e. $r_{i j}\left(T_{\mathrm{r}}\right)$ is the rain rate at time $T_{\mathrm{r}}$, expressed in $\left.\mathrm{mm} \mathrm{h}^{-1}\right)$, and $R\left(T_{k}\right)$ indicates the accumulated rain for each $T_{k}$ (e.g. for $T_{k}=00: 30, R\left(T_{k}\right)$ is the accumulated rain from 00:00 to 00:30 IDT).
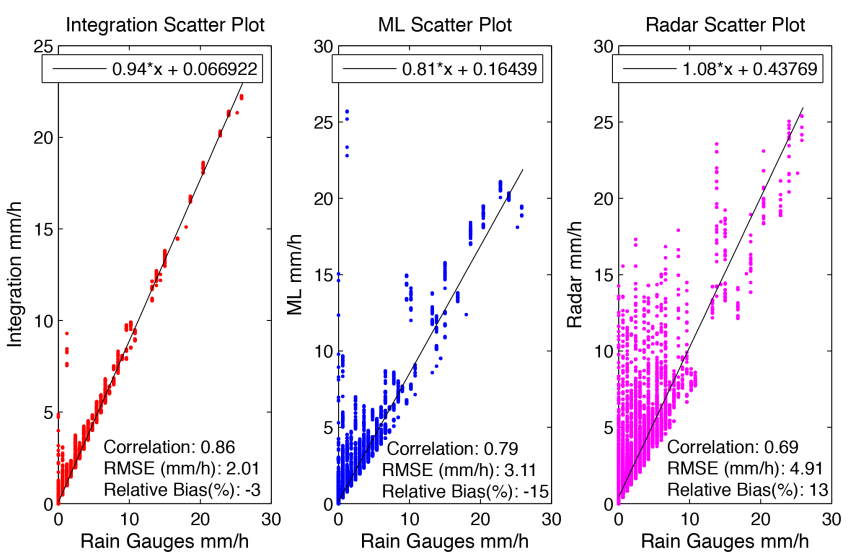

Figure 11. Rain rate scatter plots and performance evaluations of the integration (left), ML (middle) and radar (right) with respect to all the available rain gauges for event 2 .

The sites Dorot and Ramle were chosen for their respective rain events given the availability of their nearby ML data, as well as their distance from the radar. The data availability from the sources, for each site, is detailed as follows:

1. Ramle site: $\left[31.83^{\circ} \mathrm{N}, 34.96^{\circ} \mathrm{E}\right], 18$ January $2010: 24 \mathrm{~h}$ of rain; 30 operating ML in the area of interest (as shown in Fig. 13), provided by Pelephone, are available. The links operate at a frequency of $18-23 \mathrm{GHz}$ (for each link) and the link lengths vary by between 1 and $15 \mathrm{~km}$. The RSL data from the ML is given at a time resolution of 1 min with magnitude resolution of $1 \mathrm{~dB}$. Distance from the Bet Dagan radar is $17.12 \mathrm{~km}$.

2. Dorot site: $\left[31.50^{\circ} \mathrm{N}, 34.64^{\circ} \mathrm{E}\right] ; 7-10$ January 2010 , $96 \mathrm{~h}$ of rain, and 30 December 2009, $24 \mathrm{~h}$ of rain. Twelve operating ML in the area of interest (as shown 

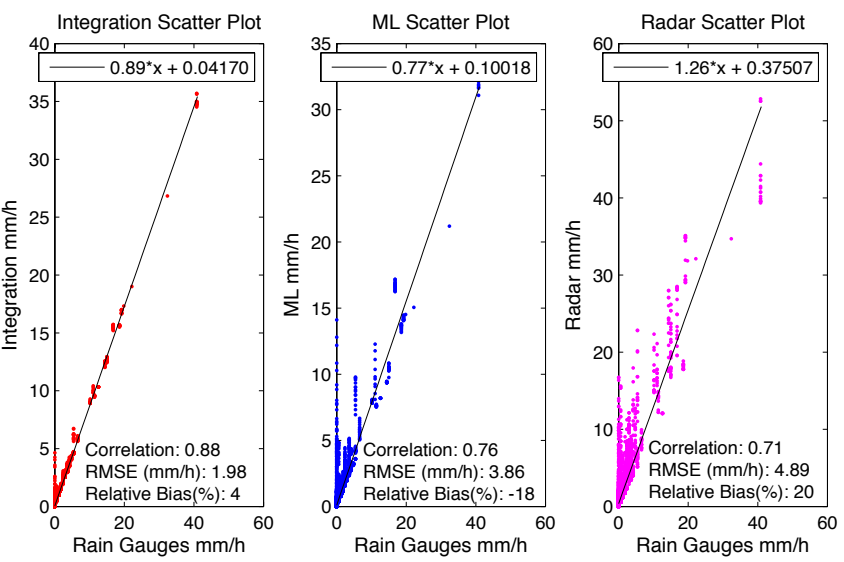

Figure 12. Rain rate scatter plots and performance evaluations of the integration (left), ML (middle) and radar (right) with respect to all the available rain gauges for event 3 .
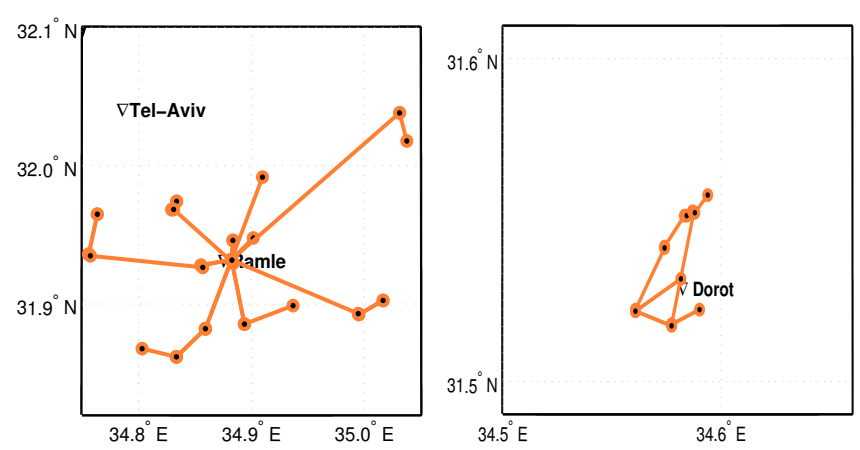

Figure 13. ML distribution for two different coordinates. Left: Ramle coordinate in an area of about $400 \mathrm{~km}^{2}$ for $30 \mathrm{ML}$ provided by Pelephone Ltd. Right: Dorot coordinate in an area of about $150 \mathrm{~km}^{2}$ for 12 ML provided by Cellcom Ltd.

in Fig. 13), provided by Cellcom Ltd., are available. The ML operate at a frequency of $17-21 \mathrm{GHz}$ (for each link) and each link length varies by between 1 and 13. Distance from the Bet Dagan radar is $53.67 \mathrm{~km}$. The RSL data from the links are given at a temporal resolution of 15 min with a magnitude resolution of $0.1 \mathrm{~dB}$.

It should be noted that we calculated the accumulated rainfall for each site over the duration for the set of events 1,2 and 3. Given that the time step varies between methods, we interpolated the accumulated results to a $5 \mathrm{~min}$ time resolution using cubic spline interpolation (De Boor, 1978) in order to make the accumulation results comparable. That is, when regarding the rain gauges for example, the time interval is $10 \mathrm{~min}$, i.e. 00:00, 00:10...23:50 IDT; thus, after interpolation, the results correspond to time samples every $5 \mathrm{~min}$, i.e. at 00:05, 00:10, 00:15..23:55 IDT.

Illustrations of the scatter plots, each with corresponding regression lines (black line), for the Ramle (event 1) and Dorot (events 2 and 3) sites are provided in Figs. 14, 15 and
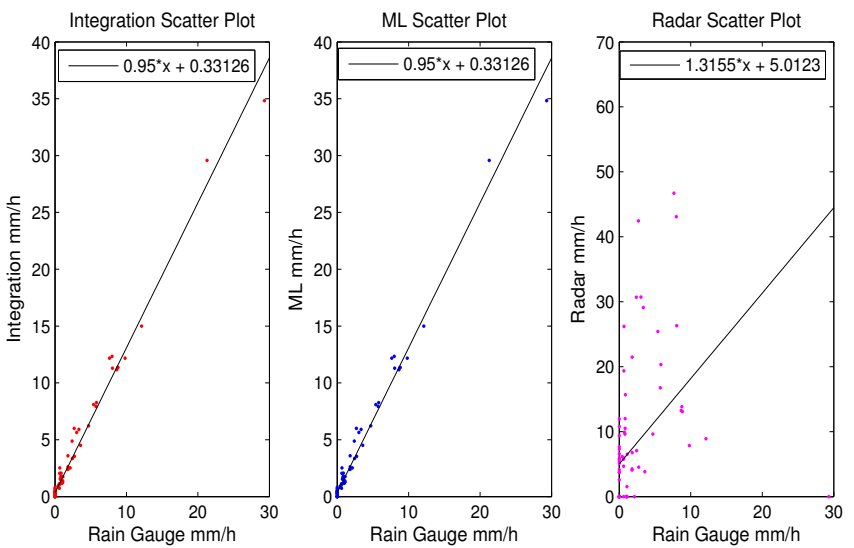

Figure 14. Rain rate scatter plots of the integration (left), ML (middle) and radar (right) with respect to the rain gauge at the Ramle site for event 1 .
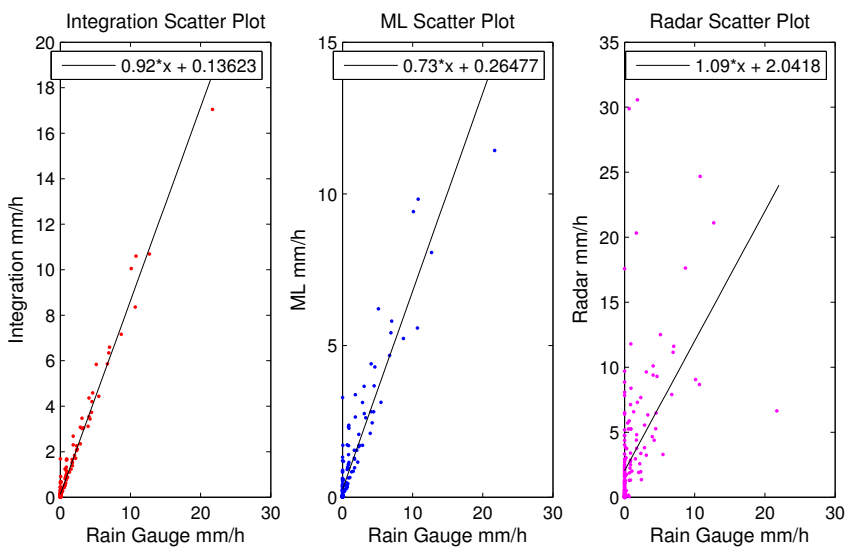

Figure 15. Rain rate scatter plots of the integration (left), ML (middle) and radar (right) with respect to the rain gauge at the Dorot site for event 2 .

16. From these figures it is clear that the integration approach achieved the lowest disparity of the points (with respect to the regression line) when comparing to the ML and the radar scatter plots. This not only implies that the integrative approach is the most accurate one but also proves the effectiveness in using Eq. (7) for the proposed integration scheme, both spatially and temporally.

Finally, Figs. 17 and 18 illustrate the accumulated rain intensity for the Ramle (event 1) and Dorot (events 2 and 3) sites. The results are demonstrated for each source, i.e. the radar (pink solid line), rain gauges (black solid line), the integration (dashed red line) and the ML (dash-dotted blue line) every 5 min (i.e. the accumulated rain during the rain event, as defined in Eq. 17).

From Figs. 17 and 18, one can see that the integration method improved the estimation of the accumulated rain, especially for the radar, which had an overestimation in all cases. When compared with ML measurements, for events 2 and 3 at Dorot, the ML showed an underestimation of the 

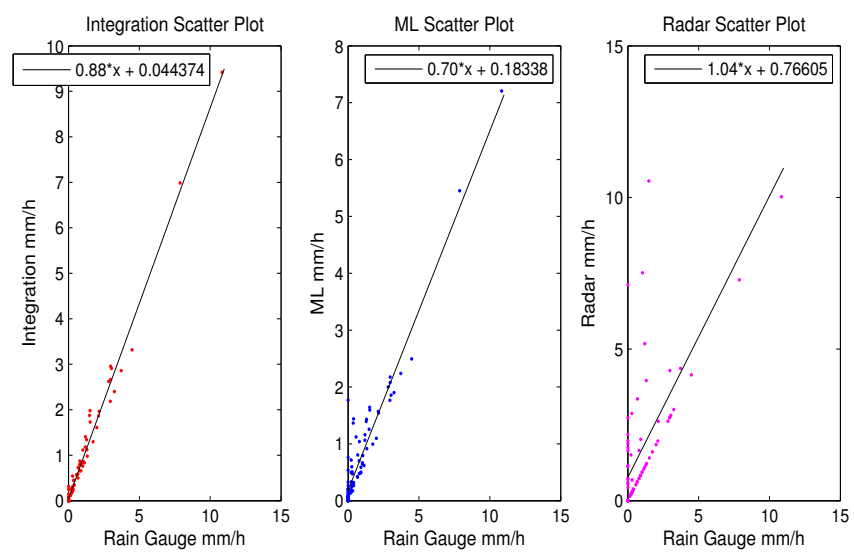

Figure 16. Rain rate scatter plots of the integration (left), ML (middle) and radar (right) with respect to the rain gauge at the Dorot site for event 3 .

Table 1. Performance analysis event (1) - Ramle site.

\begin{tabular}{llll}
\hline & Correlation & RMSE $(\mathrm{mm})$ & Relative bias $(\%)$ \\
\hline ML & 0.87 & 3.93 & -3 \\
Radar & 0.71 & 14.31 & 20 \\
Integration & 0.87 & 3.89 & -3 \\
\hline
\end{tabular}

accumulation, due to the rather sparse network deployed (only 12 available ML) in the area. On the other hand, the integrated technique provides a clear improvement. As expected, for the Ramle site there is no evident improvement in the integrated method, neither in total amounts nor with regard to the correlation or the RMSE when compared to the ML. However when comparing the performance to that of the radar, an evident improvement was clear. This is due to the high number of ML available at that point.

In order to evaluate the RMSE, RB and the spatial correlation metrics, we used Eq. (16) with $N=1$; that is, the performance measures are calculated with respect to one coordinate in space for $M$ different time steps. The evaluation is derived at the common times for all the reconstruction methods (i.e. every $30 \mathrm{~min}-00: 00,00: 30 \ldots 23: 30$ IDT) during the whole rain event. These calculations are shown for the Ramle site in Table 1, and for the Dorot site in Tables 2 and 3, with respect to events 2 and 3 . These results (and especially the RB results) of all the methods prove once again the unwavering ability of the proposed integration algorithm.

\section{Conclusions}

The ability to accurately monitor rainfall at large spatial and temporal scales is critical for meteorological and hydrological research and applications. Each of the techniques currently available (rain gauges, radar, ML and satellites) can provide important information. Each technique, however, has
Table 2. Performance analysis event (2) - Dorot site.

\begin{tabular}{llll}
\hline & Correlation & RMSE $(\mathrm{mm})$ & Relative bias (\%) \\
\hline ML & 0.77 & 6.09 & -21 \\
Radar & 0.68 & 9.19 & 19 \\
Integration & 0.88 & 2.07 & -6 \\
\hline
\end{tabular}

Table 3. Performance analysis event (3) - Dorot site.

\begin{tabular}{llll}
\hline & Correlation & RMSE $(\mathrm{mm})$ & Relative bias (\%) \\
\hline ML & 0.75 & 6.68 & -29 \\
Radar & 0.72 & 7.79 & 34 \\
Integration & 0.86 & 2.07 & 7 \\
\hline
\end{tabular}

its limitations, yet they can be used to greatly complement one another.

This paper presents a new method for data fusion of different rainfall mapping sources - the weather Radar and ML, while optimizing the advantages of each. The integration technique achieves an optimal weighted linear estimation of the rain field while considering the pros and cons of each source, mainly the coverage area of the ML and the weather radar. We have shown that the integrated approach is capable of reconstructing reliable and accurate 2-D rainfall maps compared to both spatially averaged rain gauges (Figs. 7, 8 and 9), as well as in specific locations (i.e. Figs. 17 and 18).

By using data from rain gauges from several coordinates in space, over multiple rain events, we managed to achieve an estimation for the unknown parameters in the integration model. This parameter estimation can be improved in the future as data from additional rain events become available. The main limitations of this approach lie in the necessity of having a specific model for the integration. In this paper we chose the use of a weighted linear model; the effectiveness of using this kind of model can be understood from both the scatter plots and the relative bias metric (Figs. 10, 11, 12).

The methodology proposed here is computationally fast and provides improved rainfall estimates over the entire Israel region. The data used in the analysis here show how maps can be drawn from the different sources in a manner that allows them to be compared and contrasted, as well as complementing one another, in an effort to provide a reliable assessment of the rain field recovery. The limitations are obviously the availability of data. Specifically, the ML data are subject to specific time resolution with arbitrary distribution in space, as provided by the telecommunication companies. However, once the data are accessible, we may manipulate it into uniform formats and calibrate the necessary parameters in order to provide four interdependent 2-D rainfall maps which can be used both to better inform meteorological and hydrological models as well as potentially 

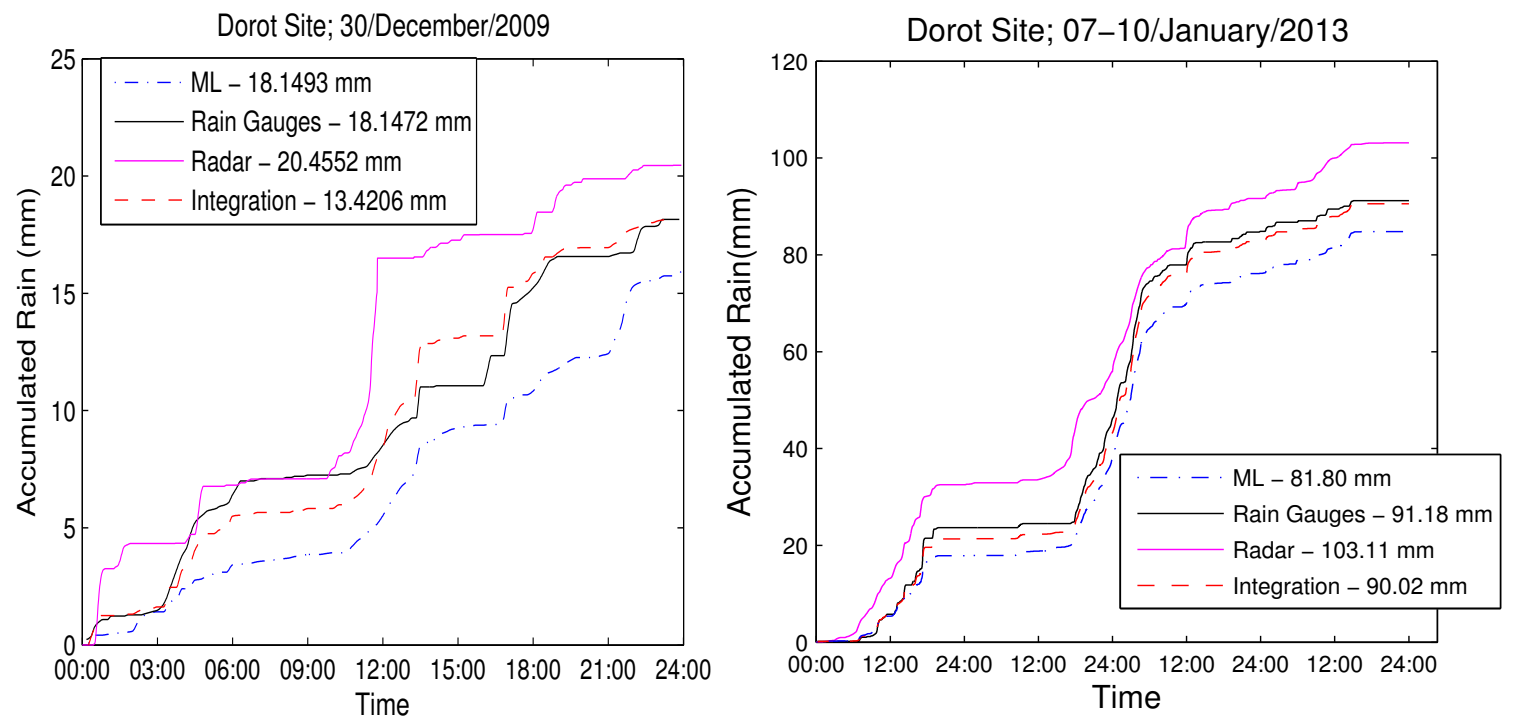

Figure 17. Accumulated rain intensity (mm) for the Dorot site, with 12 ML surrounding the site. Left: 30 December 2009 (24h of rain); right: 7-10 January 2013 (96h of rain).

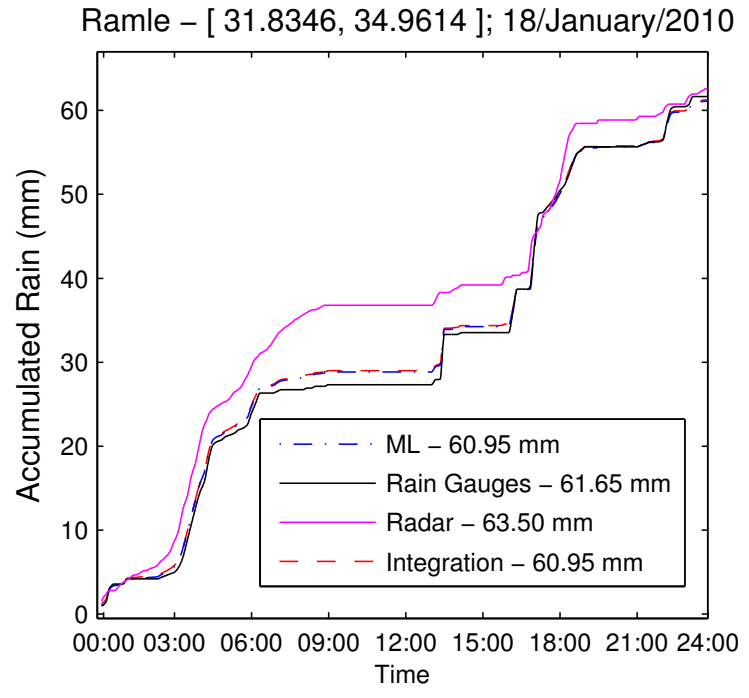

Figure 18. Accumulated rain intensity $(\mathrm{mm})$ for the Ramle site, with 30 ML surrounding the site on 18 January 2013 (24h of rain).

give a better understanding of the underlying dynamics of the storm, which no one has ever provided before.

Even though the proposed integration technique was proven to yield very accurate results, future work could focus on more complex (e.g. non-linear) models for integration between the sources, as well as the use of additional sources (e.g. satellites) in order to even achieve an improved accuracy in rainfall measurement and mapping.
Acknowledgements. The authors are deeply grateful to Y. Dagan (Cellcom Ltd.), N. Dvela and A. Shilo (Pelephone) for long-term cooperation and for providing the data for this research. We also thank the IMS for meteorological data. Partial funding for R. Samuels and P. Alpert was provided by the BSF DESERVE projects.

Edited by: J. Joiner

\section{References}

Alpert, P., Shafir, H., Cotton, W. R., and Freundlich, A.: Prediction of Meso-gamma scale orographic precipitation, Trends Hydrol., 1, 401-441, 1994.

Bringi, V. and Chandrasekar, V.: Polarimetric Doppler weather radar: principles and applications, Cambridge University Press, 2001.

Byrd, R. H., Schnabel, R. B., and Shultz, G. A.: A trust region algorithm for nonlinearly constrained optimization, SIAM J. Num. Anal., 24, 1152-1170, 1987.

Chahine, M. T., Pagano, T. S., Aumann, H. H., Atlas, R., Barnet, C., Blaisdell, J., Chen, L., Divakarla, M., Fetzer, E. J., Goldberg, M., Gautier, C., Granger, S., Hannon, S., Irion, F. W., Kakar, R., Kalnay, E., Lambrigtsen, B. H., Lee, S. Y., Le Marshall, J., and McMillan, W. W.: AIRS improving weather forecasting and providing new data on greenhouse gases., Bull. Am Meteorol. Soc., 87, 911-926, 2006.

Charvat, G. L., Williams, J., Zeng, S., and Nickalaou, J.: Small and Short-Range Radar Systems, Taylor \& Francis, 2014.

Chen, S. S., Donoho, D. L., and Saunders, M. A.: Atomic decomposition by basis pursuit, SIAM J. Sci. Comp., 20, 33-61, 1998.

Chwala, C., Gmeiner, A., Qiu, W., Hipp, S., Nienaber, D., Siart, U., Eibert, T., Pohl, M., Seltmann, J., Fritz, J., , and Kunstmann, H.: Precipitation observation using microwave backhaul links in the alpine and pre-alpine region of Southern Germany, 
Hydrol. Earth Syst. Sci., 16, 2647-2661, doi:10.5194/hess-162647-201210, 2012.

Daley, R.: Atmospheric data analysis, vol. 2, Cambridge university press, 1993.

De Boor, C.: A practical guide to splines, vol. 27, Springer-Verlag New York, 1978

Doviak, R. J., Zrnic, D. S., and Sirmans, D. S.: Doppler weather radar, Proceedings of the IEEE, 67, 1522-1553, 1979.

Eldar, Y. C.: Sampling with arbitrary sampling and reconstruction spaces and oblique dual frame vectors, J. Four. Anal. Appl., 9, 77-96, 2003.

Germann, U., Galli, G., Boscacci, M., and Bolliger, M.: Radar precipitation measurement in a mountainous region, Quart. J. Roy. Meteorol. Soc., 132, 1669-1692, 2006.

Goldshtein, O., Messer, H., and Zinevich, A.: Rain rate estimation using measurements from commercial telecommunications links, IEEE T. Signal Processing, 57, 1616- 1625, 2009.

Liberman, Y.: Optimal Recovery of Rainfall Maps Using Measurements from Wireless Sensors Network, M.Sc. Thesis, Tel Aviv University, 2013.

Liberman, Y. and Messer, H.: Accurate reconstruction of rain field maps from Commercial Microwave Networks using sparse field modeling, in: Acoustics, Speech and Signal Processing (ICASSP), 2014 IEEE International Conference on, IEEE, 67866789, 2014.

Mackenzie, A. I., Baxa Jr, E., and Harrah, S. D.: Characterization of urban ground clutter with new generation airborne Doppler weather radar, in: Radar Conference, 1993., Record of the 1993 IEEE National, IEEE, 51-56, 1993.

Marquardt, D. W.: An algorithm for least-squares estimation of nonlinear parameters, Journal of the Society for Industrial \& Applied Mathematics, 11, 431-441, 1963.

Marshall, J. S., Langille, R. C., and MCK, P. W.: Measurements of Rainfall by Radar, J. Meteorol., 4, 186-192, 1947.

McLaughlin, D.: An integrated approach to hydrologic data assimilation: interpolation, smoothing, and filtering, Adv. Water Resour., 25, 1275-1286, 2002.

Meischner, P., Bringi, V., Heimann, D., and Höller, H.: A squall line in southern Germany: Kinematics and precipitation formation as deduced by advanced polarimetric and Doppler radar measurements, Month. Weather Rev., 119, 678-701, 1991.

Messer, H., Zinevich, A., and Alpert, P.: Environmental monitoring by wireless communication networks, Science, 312, 713, 2006.
Morin, E., Krajewski, W., Goodrich, D., Gao, X., and Sorooshian, S.: Estimating rainfall intensities from weather radar data: The scale dependency problem, J. Hydrometeorol., 4, 782-797, 2003.

Morin, E., Goodrich, D. C., Maddox, R. A., Gao, X., Gupta, H. V., and Sorooshian, S.: Spatial patterns in thunderstorm rainfall events and their coupling with watershed hydrological response, Adv. Water Resour., 29, 843-860, 2006.

Olsen, R.: The $\alpha R^{\beta}$ relation in the calculation of rain attenuation, IEEE Transaction, Antenn. Propagat., 26, 318-329, 1978.

Örs, T., Chotikapong, Y., and Sun, Z.: Bisante Deliverable 2.2 :GEO Satellite Network Characteristics, 1999.

Overeem, A., Leijnse, H., and Uijlenhoet, R.: Measuring urban rainfall using microwave links from commercial cellular communication networks, Water Resour. Res., 47, W12505, doi:10.1029/WR-47-1035-2011.

Overeem, A., Leijnse, H., and Uijlenhoet, R.: Country wide rainfall maps from cellular communication networks, Proc. Natl. Ac. Sci., 110, 2741-2745, 2013.

Prat, O. P. and Barros, A. P.: Exploring the transient behavior of Z-R relationships: Implications for radar rainfall estimation, J. Appl. Meteorol. Climatol., 48, 2127-2143, 2009.

Rayitsfeld, A., Samuels, R., Zinevich, A., Hadar, U., and Alpert, P.: Comparison of two methodologies for long term rainfall monitoring using a commercial microwave communication system, Atmos. Res., 104, 119-127, 2012.

Sendik, O. and Messer, H.: On the reconstructability of images sampled by random line projections, in: Electrical \& Electronics Engineers in Israel (IEEEI), 2012 IEEE 27th Convention of, IEEE, $1-5,2012$.

Skolnik, M. I.: Introduction to Radar, Radar Handbook, 1990-1993, 1962.

Sorooshian, S., AghaKouchak, A., Arkin, P., Eylander, J., FoufoulaGeorgiou, E., Harmon, R., Hendrickx, J. M., Imam, B., Kuligowski, R., Skahill, B., and Gail, S. J.: Advancing the remote sensing of precipitation, Bull. Am. Meteorol. Soc., 92, 12711272, 2011.

Wan, E. A. and Van Der Merwe, R.: The unscented Kalman filter for nonlinear estimation, in: Adaptive Systems for Signal Processing, Communications, and Control Symposium 2000, IEEE, 153-158, 2000.

Zinevich, A., Messer, H., and Alpert, P.: Prediction of rainfall intensity measurement errors using commercial microwave communication links, Atmos. Meas. Tech., 3, 1385-1402, doi:10.5194/amt-3-1385-2010, 2010. 